\title{
Des mutations du gène de la superoxide dismutase associées à la maladie de Lou Gehrig
}

La sclérose latérale amyotrophique (SLA), qui est aussi appelé la maladie de Lou Gehrig, est un syndrome neurodégénératif qui apparaît à l'âge adulte et qui est presque toujours mortel. La probabilité actuelle de développer cette maladie est d'environ 1/1000 et l'incidence mondiale augmente. La maladie entraîne la dégénérescence des gros neurones moteurs du cerveau et de la moelle épinière. La mort de ces cellules provoque une atrophie musculaire progressive qui aboutit à une paralysie totale. Cela survient généralement entre deux et cinq ans après l'apparition des premiers symptômes. La mort des patients atteints de la SLA est habituellement due à des complications de la paralysie des muscles respiratoires. Même si, chez certains malades (5-10\%), les antécédents familiaux suggèrent une transmission héréditaire autosomale dominante, la forme la plus fréquente de cette maladie n'est pas familiale, mais sporadique. Il n'y a présentement aucun traitement pour la SLA et la cause de la maladie est complètement inconnue.

Depuis plusieurs années, deux équipes de recherche ont poursuivi l'étude de la forme familiale dans le but de déterminer la cause de la SLA et avec l'espoir de mieux comprendre la forme sporadique. Il y a deux ans, ces équipes ont déterminé qu'un gène responsable de la SLA familiale se trouvait sur le chromosome 21. Cette étude a aussi montré qu'un autre gène pourrait être responsable de près de la moitié des cas de SLA familiale; toutefois, cet autre site chromosomique n'a pas pu être localisé.

La région du chromosome 21 qui contient le gène associé à la SLA, contient aussi le gène de la superoxide dismutase 1 (SOD1), une enzyme qui pro$\mathrm{m} / \mathrm{s} n^{\circ} 5$ vol. 9, mai 93 tège les cellules contre les effets toxiques de l'oxygène en métabolisant les radicaux libres d'oxygène. Cette constatation a amené un groupe de chercheurs à yérifier l'hypothèse que le gène SOD1 puisse être responsable de la forme familiale de la SLA [1]. C'est en utilisant la technique du SSCP (single-strand conformational polymoophism) que les auteurs ont identifié 11 mutations différentes dans les exons 2 et 4 du gène SOD1 de 13 familles atteintes de SLA. Toutes ces mutations changent le codon d'un acide aminé d'une région très conservée du gène, ce qui suggère un effet sur la fonction de la superoxide dismutase. Ces observations supportent fortement la conclusion que le gène SOD1 soit responsable des formes familiales de la SLA associées au chromosome 21 .

L'association de la superoxide dismutase à la SLA suggère que les radicaux libres, qui sont normalement dégradés par cette enzyme, puissent être responsables de la pathogenèse de la mort neuronale observée dans la SLA d'origine familiale, et même dans la SLA sporadique. La toxicité des radicaux libres pourrait même être responsable de plusieurs formes de neurodégénérescence. En effet, les radicaux libres ont été impliqués dans la pathogenèse d'autres maladies neurologiques, comme la maladie de Parkinson, les lésions ischémiques cérébrales et même dans le vieillissement normal. Cette étude est la première qui démontre un rôle des radicaux libres dans une pathologie humaine.

Il reste encore plusieurs questions importantes qui sont sans réponse. Comment expliquer la sélectivité de la SLA pour les neurones moteurs, alors que la lésion génétique devrait affecter tous les tissus ? Les mutations de la SOD1 causent-elles une perte ou un gain de fonction? Il est plus probable qu'il s'agisse de mutations négatives dominantes parce que la superoxide dismutase encodée par le gène SOD1 est une enzyme active en forme de dimère. D'autre part, il est peu probable que onze mutations différentes puissent causer un gain de fonction. Quel est l'autre gène de la SLA familiale qui n'est pas lié au chromosome 21 ? La SOD1 joue-t-elle un rôle dans la pathogenèse des formes sporadiques de la SLA ?

En surcroît, cette découverte offre de nouvelles possibilités de traitement thérapeutique de la SLA. Il existe déjà plusieurs produits qui réduisent la toxicité des radicaux libres et ils pourraient être utilisés pour accroître les chances de survie des patients atteints de cette horrible maladie.

Guy Rouleau, professeur.

Centre de recherche en neurosciences, Université McGill,

1650, avenue Cedar, Montráal, Québec, H3G 1 A4.

1. Rosen DR, et al. Nature 1993 ; 362 : 59-62. 